\title{
Static hedging for knock-in/out options written on the price ratios : A simple case
}

\author{
Jirô Akahori (Research Center for Finance) \\ and \\ Katsuya Takagi (Graduate School of Mathematics) \\ Ritsumeikan University \\ 1-1-1 Nojihigashi, Kusatsu, Shiga 525-8577, Japan \\ E-mail:kenya6115@gmail.com
}

\begin{abstract}
We study static hedges of knock-in/out options written on the price ratios of several risky assets. These options are knocked-in/out when one of the prices hit against another one. The proof is given only for the simple cases.
\end{abstract}

\section{Introduction}

\subsection{Literature review}

Static hedging is a counterpart of the dynamic hedging. The word is not used for a hedge in a static model but for a less dynamic hedging in a dynamic setting.

Historically, the dynamic hedging strategy was first introduced in the celebrated Black-Scholes option pricing model [4]. Though in the paper [4], only plainvanilla (call and put) options are treated, it is now widely recognized that in the classical Black Scholes model, a path-independent type option (with some integrability) is always dynamically hedgeable by a dynamic portfolio composed of the underlying asset and the non-risky asset.

Breeden and Litzenberger [5] pointed out that any path-independent payoff can be achieved by a static portfolio of plain vanilla calls and puts. Mathematically speaking, it is based on a simple approximation argument of a function by linear combinations of $\left(x-c_{i}\right)_{+}$'s and $\left(c_{i}^{\prime}-x\right)_{+}$'s. There have been many studies (e.g. [9]) including empirical ones, about how effective the static hedges are. In the same spirit of [5] but a far more general framework was recently introduced by the first author and T. Matsusita [2]. In the paper, hedging tools are underlying risky assets that follow geometric Brownian motions as the classical Black-Scholes framework, but totally different is the amount of the risky assets. There it is assumed that the volatilities of the risky assets are very rich, and it is shown that in the situation one can statically hedges every path-independent option (with some integrability, of course).

The static hedging of knock-out/in type pathdependent options using path-independent options has been introduced in the literature by P. Carr and his co-authors [6], [7] and [8]. The strategy heavily relies on the reflection principle (see. e.g. [12]). Another application of (a kind of) reflection principle can be found in [1], where a static hedge of a path-dependent option by a combination of a path-independent option and another path-dependent option is introduced. The strategy is based on a finite-horizon version of MadanRoynette-Yor formula which associates Black-Scholes formula with the distribution function of the last exit time of the geometric Brownian motion (see for details [13] and [11]).

In the paper [3] we show that in a mild setting one can replicate the pay-off of any knock-in/out options written on several risky assets with a portfolio of pathindependent European options. The portfolio is static in the sense that we never need to change the position unless some of the prices hit against another one. This is in line with the static hedge proposed by P. Carr, and is a generalization in that the reflection principle used for the derivation is the one for multi-dimensional Brownian motions.

\subsection{Carr-Chou's static hedge}

In this section we give a brief survey on a static hedging strategy of a single barrier claim introduced by Carr and Chou [6].

They are working in a Black-Scholes economy. Namely, the price of the risky asset follows a geometric Brownian motion:

$$
S_{t}=S_{0} \exp \left(\sigma B_{t}-\frac{\sigma^{2}}{2} t+r t\right)
$$

where $B$ is a Brownian motion under the risk neutral measure, $\sigma$ is the (constant) volatility, and $r$ is the risk-free interest rate. Their method is based on the following lemma.

Lemma 1 (Carr and Chou,1997). Suppose that $X$ is a portfolio of path-independent European options expiring at time $T$ with payoff:

$$
X\left(S_{T}\right)= \begin{cases}f\left(S_{T}\right) & \text { if } S_{T} \in(A, B), \\ 0 & \text { otherwise. }\end{cases}
$$

For $H>0$, let $Y$ be a portfolio of European options 
with maturity $T$ and payoff:

$$
Y\left(S_{T}\right)= \begin{cases}\left(\frac{S_{T}}{H}\right)^{p} f\left(\frac{H^{2}}{S_{T}}\right) & \text { if } S_{T} \in\left(\frac{H^{2}}{B}, \frac{H^{2}}{A}\right), \\ 0 & \text { otherwise. }\end{cases}
$$

where the power $p \equiv 1-\frac{2 r}{\sigma^{2}}$ and $r, \sigma$ are the interest rate and volatility.

Then, $X$ and $Y$ have the same value whenever the spot equals $H$.

This lemma can be used to find the replicating portfolio of any single barrier claim. For example, consider a down-and-in claim which pays $f\left(S_{T}\right)$ at T provided a lower barrier $\mathrm{H}$ has been hit over $[0, \mathrm{~T}]$. At initiation, purchase a portfolio of European options that:

$$
\text { pay-off }= \begin{cases}0 & \text { if } S_{T}>H, \\ f\left(S_{T}\right)+\left(\frac{S_{T}}{H}\right)^{p} f\left(\frac{H^{2}}{S_{T}}\right) & \text { if } S_{T}<H .\end{cases}
$$

If the lower barrier is never hit, then the vanilla options expire worthless, matching the pay-off of zero from the down-and-in, If the barrier is hit over $[0, T]$, then Lemma 1 indicates that at the first hitting time, the value of the $\left(\frac{S_{T}}{H}\right)^{p} f\left(\frac{H^{2}}{S_{T}}\right)$ term matches the value of a pay-off $f\left(S_{T}\right) 1_{S_{T}>H}$. Thus, the options providing the pay-off $\left(\frac{S_{T}}{H}\right)^{p} f\left(\frac{H^{2}}{S_{T}}\right)$ can be sold off with the proceeds used to buy options delivering the pay-off $f\left(S_{T}\right) 1_{S_{T}>H}$. Consequently, after rebalancing at the hitting time, the total portfolio of options delivers a pay-off of $f\left(S_{T}\right)$ as required.

\subsection{The aim of the present paper}

This paper is aimed to serve as a survey of our ongoing research partly appearing in [3] on static hedging on multiple assets. The essence of the research is the use of a reflection principle of multi-dimensional situations. Simplest is the one for the multi-dimensional Brownian motion on the hyperplanes $\left\{x_{i}=x_{j}\right\}$, which principle is now extensively studied in connections with random matrices (see e.g.[10]). To be simple, we only deal with the case of three dimension, which we expect to give a good insight to the entire framework. The setting and the result is given in the first section, and the proof in the three-dimensional case is given in the latter section.

\section{Setting and the result}

\subsection{Setting}

At the beginning of this section, we give some settings. We assume that the prices of risky assets $\left\{S_{t}^{i}\right\}_{i=1, \cdots, N}$ satisfy the following condition:

$$
\frac{d S_{t}^{i}}{S_{t}^{i}}=\sigma d B_{t}^{i}
$$

where $B^{i}, i=1, \cdots, N$ are one-dimensional Brownian motions such that $d\left\langle B^{i}, B^{j}\right\rangle_{t}=0$ for $i \neq j$, and $S_{0}^{i}<$ $S_{0}^{i+1}(i=1, \cdots, N)$. Here we assume that the price of the non-risky asset is constant (in other words, the interest rate is zero), and we work on a fixed risk neutral probability measure. Under these assumptions, prices $\left\{S_{t}^{i}\right\}_{i=1, \cdots, N}$ is as follows:

$$
S_{t}^{i}=S_{0}^{i} \exp \left\{\sigma B_{t}^{i}-\frac{\sigma^{2}}{2} t\right\}
$$

Denote $S_{t}^{i, i+1}:=S_{t}^{i} / S_{t}^{i+1}(t \in(0, \infty))$, and let $\tau_{i}:=$ $\inf \left\{t \in(0, \infty): S_{t}^{i, i+1}=1\right\}$. Consider the options with the following pay-off:

$$
f\left(S_{T}^{1,2}, \cdots, S_{T}^{N-1, N}\right) 1_{\left\{\tau_{1}>T, \cdots, \tau_{N-1}>T\right\}},
$$

or

$$
f\left(S_{T}^{1,2}, \cdots, S_{T}^{N-1, N}\right) 1_{\left\{\tau_{1}<T, \cdots, \tau_{N-1}<T\right\}},
$$

where $f: \mathbb{R}^{N-1} \rightarrow \mathbf{R}$ is a continuous function with at most linear growth, and $1_{E}$ denote an indicator function of event $E$.

\subsection{A result}

Denote

$$
\begin{aligned}
& \tilde{f}\left(x_{1}, \cdots, x_{N-1}\right) \\
& \quad:=f\left(S_{0}^{1,2} \exp \left\{\sigma x_{1}\right\}, \cdots, S_{0}^{N-1, N} \exp \left\{\sigma x_{N-1}\right\}\right),
\end{aligned}
$$

and

$$
B_{t}^{i, i+1}:=B_{t}^{i}-B_{t}^{i+1}(i=1, \cdots, N-1) .
$$

With these notation, our pay-off becomes: $\tilde{f}\left(\frac{1}{\sigma} \log \frac{S_{T}^{1,2}}{S_{0}^{1,2}}, \cdots, \frac{1}{\sigma} \log \frac{S_{T}^{N-1, N}}{S_{0}^{N-1, N}}\right) 1_{\left\{\tau_{1}>T, \cdots, \tau_{N-1}>T\right\}}$.

Let

$$
R_{i}:=\left\{S_{T}^{i, i+1} \in(0,1)\right\}(i=1, \cdots, N-1),
$$

and for the set $A \subset\{1, \cdots, N-1\}$, define a function

$$
\tilde{f}_{A}\left(x_{1}, \cdots, x_{N-1}\right):=\tilde{f}\left(y_{1}, \cdots, y_{N-1}\right),
$$

where

$$
y_{i}= \begin{cases}x_{i} & i \notin A, \\ \frac{2}{\sigma} \log \frac{1}{S_{0}^{i, i+1}}-x_{i} & i \in A .\end{cases}
$$

Theorem 2. The following strategy statically hedges the option with pay-off (1):

1 At initiation, purchase a portfolio of European options that:

$$
\begin{array}{ll}
\text { pay-off } & \\
= & (-1)^{\# A} \tilde{f}_{A}\left(\frac{1}{\sigma} \log \frac{S_{T}^{1,2}}{S_{0}^{1,2}}, \cdots, \frac{1}{\sigma} \log \frac{S_{T}^{N-1, N}}{S_{0}^{N-1, N}}\right) \\
& \cdot 1_{\left\{{ }_{i \in A} R_{i}^{c}\right\} \cap\left\{\bigcap_{i \notin A} R_{i}\right\}},
\end{array}
$$

where $A \subset\{1, \cdots, N-1\}, R_{i}^{c}=\Omega \backslash R_{i}$, and $\# A:=\{$ number of elements belonging to $A\}$. 
2 If some two prices $S^{k}$ and $S^{k+1}(k=1, \cdots, N-$ 1) are touched at time $\tau:=\min \left(\tau_{1}, \cdots, \tau_{N-1}\right)$ before maturity $T$, then at the time $\tau$, for any $C \subset\{1, \cdots, k-$ $1, k+1, \cdots, N-1\}$, the value of

$$
\begin{array}{r}
(-1)^{p} \tilde{f}_{C}\left(\frac{1}{\sigma} \log \frac{S_{T}^{1,2}}{S_{0}^{1,2}}, \cdots, \frac{1}{\sigma} \log \frac{S_{T}^{N-1, N}}{S_{0}^{N-1, N}}\right) \\
\cdot 1_{\left\{{ }_{i \in C} \cap R_{i}^{c}\right\} \cap\left\{{ }_{i \notin C} R_{i}\right\} \cap R_{k}},
\end{array}
$$

where $p:=\# C$, matches the value of

$$
\begin{array}{r}
-(-1)^{\tilde{p}} \tilde{f}_{C \cup\{k\}}\left(\frac{1}{\sigma} \log \frac{S_{T}^{1,2}}{S_{0}^{1,2}}, \cdots, \frac{1}{\sigma} \log \frac{S_{T}^{N-1, N}}{S_{0}^{N-1, N}}\right) \\
\left.\cdot 1_{\{i \in C} R_{i}^{c}\right\} \cap\left\{{ }_{i \notin C} R_{i}\right\} \cap R_{k}^{c},
\end{array}
$$

where $\tilde{p}:=\#\{C \cup\{k\}\}$. Thus, the options providing the pay-off (2) can be sold off with the proceeds used to buy options delivering the pay-off (3).

3 Consequently, after rebalancing at time $\tau$, the total portfolio of options delivers a pay-off of 0 . If any pair of prices is not touched, this portfolio will be worth (4) at maturity $T$ :

$$
\begin{array}{r}
(-1)^{\# \phi} \tilde{f}_{\phi}\left(\frac{1}{\sigma} \log \frac{S_{T}^{1,2}}{S_{0}^{1,2}}, \cdots, \frac{1}{\sigma} \log \frac{S_{T}^{N-1, N}}{S_{0}^{N-1, N}}\right) \\
=f\left(S_{T}^{1,2}, \cdots, S_{T}^{N-1, N}\right) .
\end{array}
$$

The proof is almost strait-forward if we admit the following lemma.

Lemma 3. If $\tau_{k}=\min \left(\tau_{i}: i=1, \cdots, N-1\right)<T$, then for any $C \subset\{1, \cdots, k-1, k+1, \cdots, N-1\}$, the value of

$$
\begin{array}{r}
(-1)^{p} \tilde{f}_{C}\left(\frac{1}{\sigma} \log \frac{S_{T}^{1,2}}{S_{0}^{1,2}}, \cdots, \frac{1}{\sigma} \log \frac{S_{T}^{N-1, N}}{S_{0}^{N-1, N}}\right) \\
1_{\left\{{ }_{i \in C} \cap R_{i}^{\mathrm{c}}\right\} \cap\left\{{ }_{i \notin C} R_{i}\right\} \cap R_{k}},
\end{array}
$$

where $p:=\# C$, matches the value of

$$
\begin{aligned}
& -(-1)^{\bar{p}} \tilde{f}_{C \cup\{k\}}\left(\frac{1}{\sigma} \log \frac{S_{T}^{1,2}}{S_{0}^{1,2}}, \cdots, \frac{1}{\sigma} \log \frac{S_{T}^{N-1, N}}{S_{0}^{N-1, N}}\right) \\
& \cdot 1\left\{\bigcap_{i \in C} R_{i}^{c}\right\} \cap\left\{\bigcap_{i \notin C} R_{i}\right\} \cap R_{k}^{c},
\end{aligned}
$$

where $\tilde{p}:=\#\{C \cup\{k\}\}$.

Proof of this lemma in the case of $\mathrm{N}=3$ will be given in the next section.

\section{Static hedging strategy and Proof of Lemma 3 in the case of $\mathrm{N}=3$}

We prove Lemma 3 only for the case of $N=3$. For the general cases, see [3].

In this case, our pay-off is following:

$$
\text { pay-off }=f\left(S_{T}^{1,2}, S_{T}^{2,3}\right) 1_{\left\{\tau_{1}>T, \tau_{2}>T\right\}},
$$

This equation means that: If some pair $\left(S^{1}, S^{2}\right)$ or $\left(S^{2}, S^{3}\right)$ is touched (Fig.1), We gain nothing, and otherwise (Fig.2), We gain $f\left(S^{1,2}, S^{2,3}\right)$.

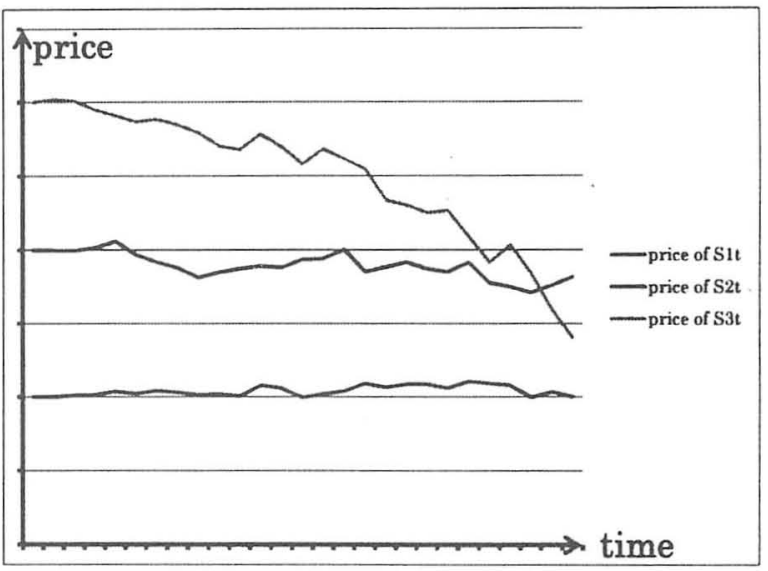

Fig. 1: Hitting case

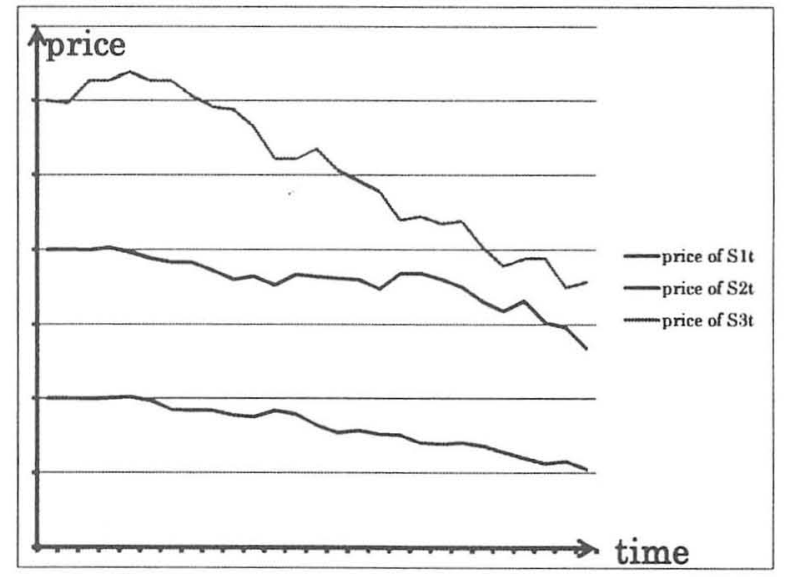

Fig. 2: No-hitting case

In this case, our static hedging strategy is as follows: 
At the initial time, purchase a portfolio of European options with pay-off $=$

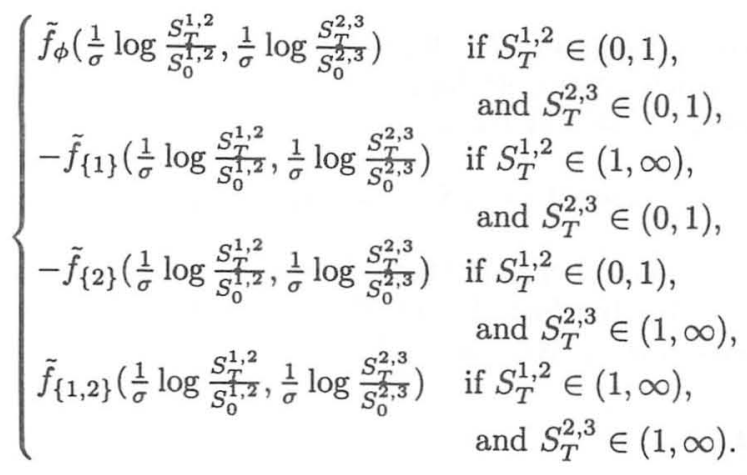

Lemma 3 states that: If $\tau_{1}=\min \left(\tau_{1}, \tau_{2}\right)<T$, At time $\tau_{1}$ the value of

$$
\tilde{f}_{\phi}\left(\frac{1}{\sigma} \log \frac{S_{T}^{1,2}}{S_{0}^{1,2}}, \frac{1}{\sigma} \log \frac{S_{T}^{2,3}}{S_{0}^{2,3}}\right) 1_{\left\{S_{T}^{1,2} \in(0,1), S_{T}^{2,3} \in(0,1)\right\}},
$$

matches the value of

$$
\tilde{f}_{\{1\}}\left(\frac{1}{\sigma} \log \frac{S_{T}^{1,2}}{S_{0}^{1,2}}, \frac{1}{\sigma} \log \frac{S_{T}^{2,3}}{S_{0}^{2,3}}\right) 1_{\left\{S_{T}^{1,2} \in(1, \infty), S_{T}^{2,3} \in(0,1)\right\}},
$$

and the value of

$$
-\tilde{f}_{\{2\}}\left(\frac{1}{\sigma} \log \frac{S_{T}^{1,2}}{S_{0}^{1,2}}, \frac{1}{\sigma} \log \frac{S_{T}^{2,3}}{S_{0}^{2,3}}\right) 1_{\left\{S_{T}^{1,2} \in(0,1), S_{T}^{2,3} \in(1, \infty)\right\}},
$$

matches the value of

$$
-\tilde{f}_{\{1,2\}}\left(\frac{1}{\sigma} \log \frac{S_{T}^{1,2}}{S_{0}^{1,2}}, \frac{1}{\sigma} \log \frac{S_{T}^{2,3}}{S_{0}^{2,3}}\right) 1_{\left\{S_{T}^{1,2} \in(1, \infty), S_{T}^{2,3} \in(1, \infty)\right\}} .
$$

In other words,

$$
\mathbf{E}\left[\text { the quantity }(7) \mid \mathcal{F}_{\tau_{1}}\right]=\mathbf{E}\left[\text { the quantity }(8) \mid \mathcal{F}_{\tau_{1}}\right]
$$

and

$\mathbf{E}\left[\right.$ the quantity $\left.(9) \mid \mathcal{F}_{\tau_{1}}\right]=\mathbf{E}\left[\right.$ the quantity $\left.(10) \mid \mathcal{F}_{\tau_{1}}\right]$.

Here $\left\{\mathcal{F}_{t}\right\}$ is the natural filtration of the Brownian motions $\left(B^{1}, B^{2}\right)$, and $\mathcal{F}_{\tau}$ for any stopping time $\tau$ is defined as usual (see e.g. [12]).

Thus, we noticed that to prove Lemma 3 , in the case of $N=3$ is equivalent to establishing the identities (11) and (12). We can express the quantity in the left-handside of (11) as

$$
\begin{aligned}
& \mathbb{E}\left[\text { the quantity }(7) \mid \mathcal{F}_{\tau_{1}}\right] \\
& =\int_{\left\{S_{T}^{1,2} \in(0,1), S_{T}^{2,3} \in(0,1)\right\}} \tilde{f}_{\phi}\left(B_{T}^{1,2}, B_{T}^{2,3}\right) d P .
\end{aligned}
$$

We can rewrite this equation by using the jointprobability density function $\varphi\left(x_{1}, x_{2}\right)$ of $\left(B_{T}^{1,2}, B_{T}^{2,3}\right)$ :

$\varphi\left(x_{1}, x_{2}\right):=\frac{1}{2 \pi b_{1} b_{2}} \exp \left\{-\frac{1}{2}\left(\frac{\left(x_{1}-a_{1}\right)^{2}}{\left(b_{1}\right)^{2}}+\frac{\left(x_{2}-a_{2}\right)^{2}}{\left(b_{2}\right)^{2}}\right)\right\}$, where $a_{i}:=B_{\tau_{1}}^{i, i+1}, b_{i}:=\left(V\left(B_{T}^{i, i+1} \mid B_{\tau_{1}}^{i, i+1}\right)\right)^{1 / 2}(i=$ $1,2)$. By using the density,

$$
\begin{aligned}
& \mathbf{E}\left[\text { the quantity (7) } \mid \mathcal{F}_{\tau_{1}}\right]
\end{aligned}
$$

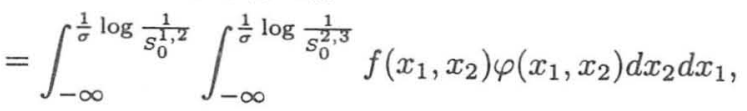

and

$$
\begin{aligned}
& \mathbf{E}\left[\text { the quantity }(8) \mid \mathcal{F}_{\tau_{1}}\right] \\
& =-\int_{\frac{1}{\sigma} \log \frac{1}{S_{0}^{1,2}}}^{\infty} \int_{-\infty}^{\frac{1}{\sigma} \log \frac{1}{S_{0}^{2,3}} \varphi\left(x_{1}, x_{2}\right)} \\
& \cdot f\left(\frac{2}{\sigma} \log \frac{1}{S_{0}^{1,2}}-x_{1}, x_{2}\right) d x_{2} d x_{1} .
\end{aligned}
$$

In the equation (13), put $y=\frac{2}{\sigma} \log \frac{1}{S_{0}^{1,2}}-x_{1}$, then

$$
\begin{aligned}
& \left.\mathbf{E} \text { [the quantity (8) } \mid \mathcal{F}_{\tau_{1}}\right]
\end{aligned}
$$

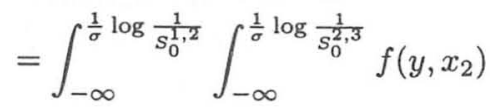

$$
\begin{aligned}
& \cdot \varphi\left(\frac{2}{\sigma} \log \frac{1}{S_{0}^{1,2}}-y, x_{2}\right) d x_{2} d y .
\end{aligned}
$$

Now it suffices to show that

$$
\varphi\left(\frac{2}{\sigma} \log \frac{1}{S_{0}^{1,2}}-y, x_{2}\right)=\varphi\left(y, x_{2}\right)
$$

This is in fact almost direct by the expression

$\varphi\left(x_{1}, x_{2}\right)=\frac{1}{2 \pi b_{1} b_{2}} \exp \left\{-\frac{1}{2}\left(\frac{\left(x_{1}-a_{1}\right)^{2}}{\left(b_{1}\right)^{2}}+\frac{\left(x_{2}-a_{2}\right)^{2}}{\left(b_{2}\right)^{2}}\right)\right\}$,

where $a_{1}=B_{\tau_{1}}^{1,2}=\frac{1}{\sigma} \log \frac{1}{S_{0}^{1,2}}$.

Similarly, we can show the identity (12).

Financially speaking, this means that the options providing the pay-off (7) and (9) can be sold off with the proceeds used to buy the options delivering the pay-off (8) and (10).

If $\tau_{2}=\min \left(\tau_{1}, \tau_{2}\right)<T$, we can get a similar result.

Consequently, after rebalancing at time $\tau_{1}$, the total portfolio of options delivers a pay-off of 0 . If any pair of prices is not touched, this portfolio will be worth $f\left(S_{T}^{1,2}, S_{T}^{2,3}\right)$.

\section{References}

[1] Akahori, J. Imamura, Y. and Yano, Y. (2009) "On the Pricing of Options Written on the Last Exit Time", Methodology and Computing in Applied Probability, vol. 11 no.4, pp661-668.

[2] Akahori, J. and Matsusita, T. (2010) "Static Hedges in Supermarkets", preprint, Ritsumeikan Universily. 
[3] Akahori, J. and Takagi, K. (2010) "Static Hedging for knock-in/out options written on multiple assets", preprint, Ritsumeikan University.

[4] Black, F. and Scholes, M. (1973) "The Pricing of Options and Corporate Liabilities", Journal of Political Economy, vol. 81, pp637-654.

[5] Breeden, D. and Litzenberger, R. (1978) "Prices of State Contingent Claims Implicit in Option Prices", Journal of Business, 51, 621-651.

[6] Carr, P. and Chou, A. (1997) "Breaking Barriers: Static Hedging of Barrier Securities", Risk, Vol.10, No.9, 139-145.

[7] Carr, P., Ellis, K. and Gupta, V. (1998), "Static Hedging of Exotic Options", Journal of Finance, vol. 53 , pp1165-1190.

[8] Carr, P. and Picron, J.F. (1999) "Static Hedging of Timing Risk", The Journal of Derivatives, vol. 6, no. 3: pp. 57-70.

[9] Derman, E. Ergener, D. and Kani, I. (1995) "Static Options Replication" The Journal of Derivatives, vol. 2, no. 4: pp. 78-95.

[10] Grabiner, D.J. (1999), "Brownian Motion in a Weyl Chamber, Non-Colliding Particles, and Random Matrices",Ann. Inst. H. Poincare Probab. Statist. vol. 35 , no. 2, pp177-204.

[11] Imamura, Y. (2010) "A Remark on Static Hedging of Options Written on Last Exit Time", preprint, Ritsumeikan University.

[12] Karatzas, I. and Shreve, S. (1992), Brownian Motion and Stochastic Calculus, 2nd eds. SpringerVerlag.

[13] Madan, D., Roynette, B., and Yor. M. (2008) "Unifying Black-Scholes Type Formulae which Involve Last Passage Times up to Finite Horizon" AsiaPacific Financial Markets, vol.15, no.2, pp97-115. 